\title{
Cervical Myelopathy Secondary to Atlanto-occipital Assimilation: The Usefulness of the Simple Decompressive Surgery
}

\author{
Kang Rae Kim, Young Min Lee, Young Zoon Kim, Yong Woon Cho, \\ Joon Soo Kim, Kyu Hong Kim, In Chang Lee \\ Department of Neurosurgery, Samsung Changwon Hospital, Sungkyunkwan University School of Medicine, Changwon, Korea
}

Atlanto-occipital assimilation is one of the most common osseous anomalies observed at the craniocervical junction. Most patients with atlas assimilation show no symptom, but some have neurological problems such as myelopathy that may require surgical treatment. Occipitocervical fusion may be required if atlato-occipital assimilation is accompanied by occipito-axial instability. However, in cases of symptomatic atlas assimilation with minor cord compression without instability, simple decompressive surgery may be the treatment modality. This report describes a case of successful treatment of a patient with myelopathy secondary to atlanto-occipital assimilation without instability, using posterior simple decompressive surgery.

Key Words: Atlanto-occipital joint $\cdot$ Assimilation $\cdot$ Myelopathy $\cdot$ Decompression

\section{INTRODUCTION}

Craniocervical abnormalities have been reported in various studies for many years. Atlas occipitalization or assimilation is one of the most common osseous congenital anomalies of the craniovertebral junction ${ }^{5}$. Atlas assimilation refers to the congenital fusion of the atlas with the base of the occipital bone. According to Yochum and Rowe ${ }^{12)}$, occipitalization represents the most cephalic 'blocked' vertebra encountered in the spine.

Some patients with atlas assimilation may be asymptomatic, and others may experience many serious symptoms, from neural compression caused either by encroachment into the foramen magnum or posterior arch projection into the spinal canal.

For proper neural decompression or decompression with fusion, many surgical procedures can be considered in those patients. For patients with instability, occipitocervical fusion surgery should be performed. For patients without instability, however, posterior simple decompressive surgery could be a treatment modality. The suitability of various surgical treat-

\footnotetext{
- Received: June 20, 2013 - Revised: August 27, 2013

- Accepted: August 29, 2013

Corresponding Author: Young Min Lee, MD

Department of Neurosurgery, Samsung Changwon Hospital, Samsung

Medical Center, Sungkyunkwan University School of Medicine, 158

Paryong-ro, Masanhoiwon-gu, Changwon 630-723, Korea

Tel: +82-55-290-6183, Fax: +82-55-290-6899

E-mail: mulsae@hanmail.net
}

ment options should be evaluated on a case-by-case basis.

The following case report illustrates that even a simple surgical procedure can be an effective form of treatment for some patients with atlas assimilation.

\section{CASE REPORT}

A 36-year-old male presented himself with a one-month history of numbness of all his fingertips in his left hand, hypesthesia, posterior neck pain, and left shoulder pain. The neurological examination upon his admission revealed marked restriction in the range of the left forward bending motion of his neck due to an electrical sensation that was running down his back and into his limbs (Lhermitte's sign) and that included a positive Hoffmann's sign.

Cervical plain film radiography and computed tomography (CT) revealed partial circumferential fusion of the anterior and posterior $\mathrm{C} 1$ arches with the basilar part of the occipital bone (complete anterior fusion and partial, asymmetrical posterior fusion) and incomplete block vertebrae on C2-3 (C2 vertebral arch and C3 lamina fusion on the left side) (Fig. 1). Cervical magnetic resonance imaging (MRI) revealed high signal intensity in the spinal cord on the T2 weighted images, which indicates spinal distress caused by the encroachment of the left $\mathrm{C} 1$ posterior arch and the left $\mathrm{C} 2$ vertebral arch on the neural canal (Fig. 2). The patient's symptoms were regar ded as the consequence of the encroachment of the bony element on the neural canal. There was also no occipitocervical 

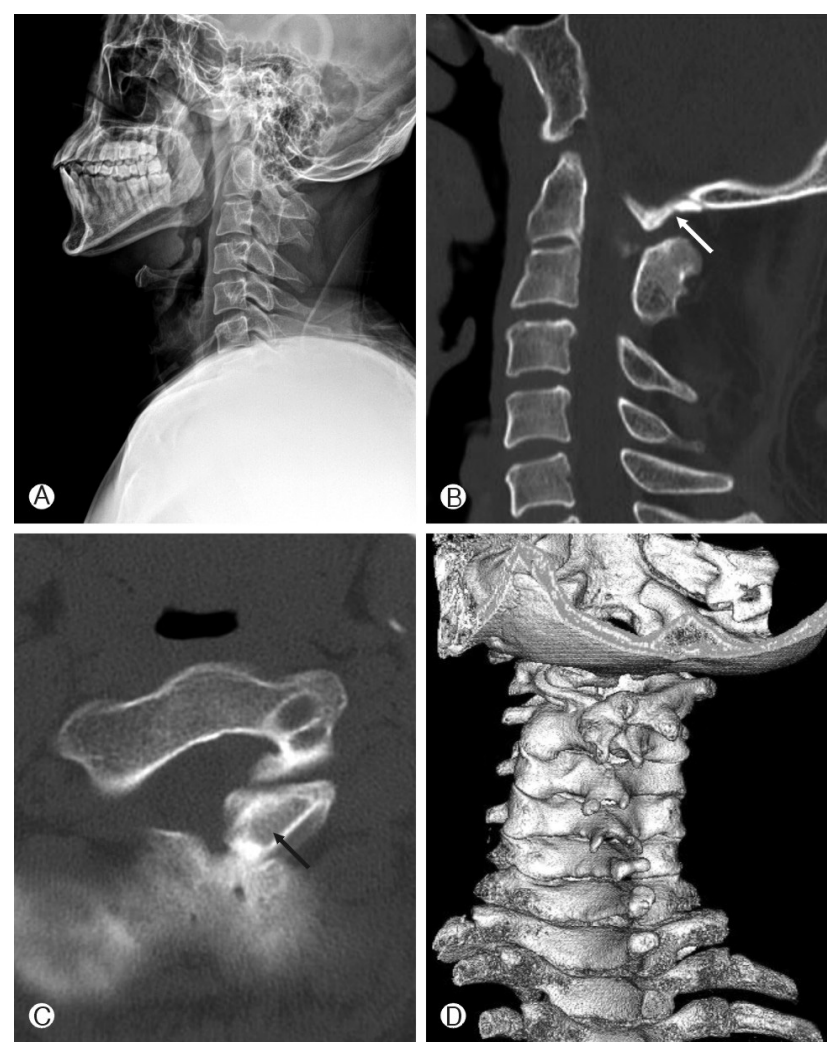

Fig. 1. Preoperative lateral radiograph (A), sagittal, left side (B), axial (C) and 3-demensional (3D) (D) computed tomography reveal atlanto-occipital assmilation, thecal sac compression by $\mathrm{Cl}, 2$ posterior arch encroachment (arrow) and C2-3 fusion on posterior element.

instablility. Thus, the patient underwent posterior surgery for partial removal of his C1 left posterior arch and his C2 left vertebral arch to decompress his thecal sac.

A day after the surgery, the numbness of the patient's fingertips, his hypesthesia, and Lhermitte's sign disappeared. The image of the decompression of the thecal sac was confirmed with a post-operative CT scan (Fig. 3). The absence of post-operative instablility was also confirmed.

\section{DISCUSSION}

Atlas assimilation is one of the most common congenital osseous malformations of the cranio-cervical junction ${ }^{5}$. It is estimated to affect $0.08-3 \%$ of the general population ${ }^{2,11}$. The fusion of the atlas with the occipital bone constitutes the anomaly.

In the embryonic period, the somites are differentiated into sclerotomes on the fourth week of gestation. The caudal half of each sclerotome combines with the rostral half of the sclerotome below it to form one vertebra each ${ }^{4,8)}$. Atlanto-occipital

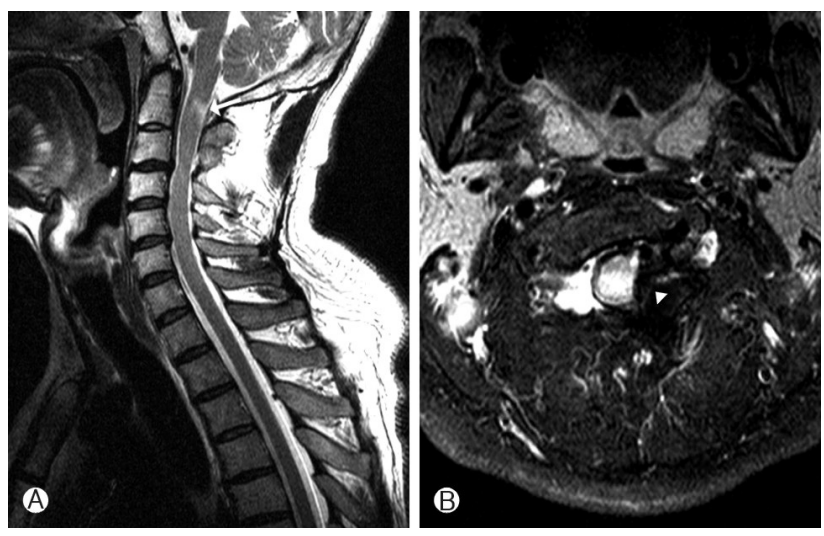

Fig. 2. Sagittal (A) and axial (B) T2-weighted magnetic resonance imaging reveal high signal intensity (arrow) within the spinal cord on cranio-cenvial junction and compressed thecal sac on left side (arrow head).
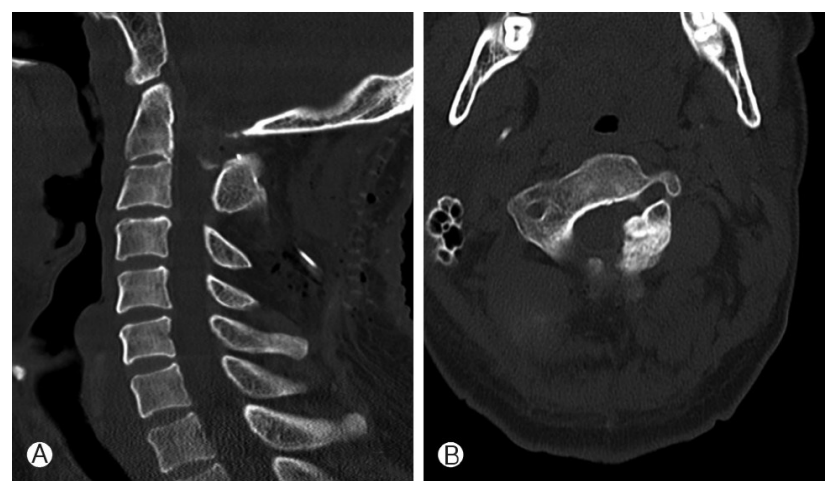

Fig. 3. Postoperative sagittal (A), axial (B) computed tomography reveal thecal sac decompression by partial removal of left posterior arch of $\mathrm{Cl}, 2$.

assimilation may happen occasionally in case of disruption of the segmentation and separation of the caudal occipital sclerotome and the rostral cervical sclerotome. This condition may be complete or partial ${ }^{10)}$, with the latter being the most common $^{2,11)}$. In atlas assimilation, the spinal canal may be anteriorly compressed by fibrosis in the retro-odontoid space or posteriorly by a partially assimilated posterior arch, and so on.

Patients with cranio-cervical junction anomalies often do not present neurological signs and symptoms before the age of 20. Atlas assimilation may be asymptomatic, but various signs and symptoms can occur in patients afflicted with it. These include headache, neck pain, numbness and pain in the extremities, weakness, ataxia, and symptoms connected to vertebral artery compression, which may each occur alone or with those of the spinal cord compression ${ }^{1)}$. In the patient in this case study, the partially assimilated posterior arch of the atlas compressed the spinal cord, which produced myelopathy.

Many surgical options can be considered for symptomatic 
patients. Jain et al. ${ }^{3)}$ have described the technique of drilling the occipital bone close to the foramen magnum and forming an artificial arch of the atlas, which is subsequently used for atlantoaxial fixation. Occipital wiring, hook fixation, and rigid fixation can all be surgical options, but rigid fixation with occipital screws seems superior to wiring-based stabilization methods ${ }^{7,9)}$. Among the many surgical options, occipito-cervical fusion surgery should be performed for patients with a narrowed spinal canal and instability. Posterior decompression and fusion surgery, including laminectomy of the $\mathrm{C} 1$ and occipitocervical arthrodesis at $\mathrm{C} 2-3$, could be considered. Because atlanto-occipital assimilation is frequently accompanied by a congenital anomaly in the other cervical spine, the surgical approach should be tailored to individual patients, especially with screw fixation. Simple decompressive surgery for atlanto-occipital assimilation has rarely been reported. For symptomatic patients with a narrowed spinal canal and minor cord compression without instability, posterior simple decompressive surgery such as laminectomy could be an effective form of treatment. Posterior simple decompressive surgery can also reduce the post-operative morbidity that may happen in occipito-cervical fusion surgery. However, when only decompressive surgery is performed, because wide laminectomy can cause spinal instability, the extent of the laminectomy should be as small as possible, as in the case in this study. Moreover, it must also be checked if post-operative instability happened or not after simple decompressive surgery.

\section{CONCLUSION}

Many variable types of atlas assimilation have been reported. Atlas assimilation may include alteration of the atlantooccipital articulation or narrowing of the spinal canal. Most of the abnormalities cause no typical symptom, but some patients have neurological problems. Patients with neurologic symptoms caused by minor posterior compression of the spinal cord without instability can be treated successfully with posterior simple decompressive surgery.

\section{REFERENCES}

1. Hensinger RN: Osseous anomalies of the craniovertebral junction. Spine 11:323-333,1986

2. Iwata A, Murata M, Nukina N, Kanazawa I: Foramen magnum syndrome caused by atlnato-occipital assimilation. J Neurol Sci 154:229-231,1998

3. Jain VK, Takayasu M, Singh S, Chharbra DK, Sugita K: Occipital-axis posterior wiring and fusion for atlantoaxial dislocation associated with occipitalization of atlas: technical note. J Neurosurg 79:142, 1993

4. Kaplan KM, Spivak JM, Bendo JA: Embryology of the spine and associated congenital abnormalities. Spine J 5:564-576, 2005

5. Lang J: Skull base and related structures, Atlas of Clinical Anatomy, Newyork: Shattauer, Stutgart pp 54, 1995

6. Moore K, Persaud TVN: The developing human: clinically oriented embryology, ed6. W.B. Saunders Company, Philadelphia, 1998

7. Pait TG, Al-Mefty O, Boop FA, Arnautovic KI, Rahman S, Ceola $\mathrm{W}$ : Inside-outside technique for posterior occipitocervical spine instrumentation and stabilization: preliminary results. J Neurosurg 90(1 Suppl):1-7,1999

8. Sadler TW, Langman J: Medical embryology, ed8. Pradel, 2007

9. Sandhu FA, Pait TG, Benzel E, Henderson FC: Occipitocervical fusion for rheumatoid arthritis using the inside-outside stabilization technique. Spine 28:414-419,2003

10. Tun K, Okutan O, Kaptanoglu E, Gok B, Solaroglu I, Beskonakli E: Inverted hypertrophy of occipitalcondyles associated with atlantooccipital fusion and basilar invagination: a case report. Neuroanatomy 3:43-45, 2004

11. Wackenhein A: Roentgen diagnosis of the cranio vertebral region. New York: Springer-Verlag:360-362, 1974

12. Yochum TR, Rowe LJ: Essentials of skeletal radiology, ed2. Baltimore: William and Wilkins, Vol 1, pp3, 1987 